\title{
GASTROPOD MOLLUSKS FROM THE CONTINENTAL SHELF OFF JALISCO AND COLIMA, MEXICO: SPECIES COLLECTED WITH A TRAWL NET
}

\section{MOLUSCOS GASTRÓPODOS DE LA PLATAFORMA CONTINENTAL DE JALISCO Y COLIMA, MÉXICO: ESPECIES RECOLECTADAS CON RED DE ARRASTRE}

\author{
Martín Pérez-Peña \\ Eduardo Ríos-Jara* \\ Laboratorio de Ecosistemas Marinos y Acuicultura, Departamento de Ecología \\ Centro Universitario de Ciencias Biológicas y $\Lambda$ gropecuarias \\ Universidad de Guadalajara \\ Apartado postal 52-114 \\ Zapopan, CP 45030, Jalisco, México \\ *E-mail: cdurios@maiz.cucba.udg.mx
}

Recibido en noviembre de 1997: aceptado en septiembre de 1998

\begin{abstract}
This study examines the distribution and abundance with respect to depth and type of substratum of 86 gastropod species collected from the Pacific continental shelf off Jalisco and Colima, Mexico, in August 1988. Sampling was performed with a trawl net at 22 stations, al depths of 18 to $112 \mathrm{~m}$. A total of 582 individuals pertaining to 42 genera, 25 families, 4 orders and 3 subclasses of gastropods were collected. Abundance of gastropods was similar between 18 and $83 \mathrm{~m}$, but the number of live individuals decreased between 41 and $60 \mathrm{~m}$. Deep $(61-83 \mathrm{~m})$ stations registered the lowest diversity; however, the number of species collected alive decreased with depth. No gastropods were collected at stations deeper than $83 \mathrm{~m}$. The number of individuals and species also varied with the type of substratum. Greater heterogeneity of the sediments was found at the shallower stations $(18-60 \mathrm{~m})$ with medium sand, sandy silt and silty clay substrata. The greatest number of species were collected at stations with sandy silt and medium sand substrata. Total abundance was notably smaller in medium sand, while the abundance of live gastropods was higher in silty clay substratum. The seven most abundant species represented $51.2 \%$ of all of the individuals. Most of these species are of commercial interest, in particular those of the genera Fusinus, Hexaplex, Ficus, Harpa, Cantharus and Bursa.
\end{abstract}

Key words: gastropods, benthos, continental shelf, Mexican Pacific.

\section{RESUMEN}

En este estudio se examina la distribución y abundancia con respecto a la profundidad y tipo de substrato de 86 especies de gastrópodos recolectados en la plataforma continental de lalisco y Colima, México, en agosto de 1988. El muestreo se realizó con una red de arrastre en 22 estaciones, a profundidades de entre 18 y $112 \mathrm{~m}$. Se recolectaron un total de 582 individuos pertenecientes a 42 géneros, 25 familias, 4 órdenes y 3 subclases de gastrópodos. La abundancia de los gastrópodos fue similar entre 
los 41 y $83 \mathrm{~m}$, aunque el número de individuos vivos disminuyó entre los 41 y $60 \mathrm{~m}$. Las estaciones profundas $(6 \mathrm{l}-83 \mathrm{~m})$ registraron la menor diversidad; sin emhargo, el número de especies recolectadas vivas disminuyó con la profundidad. Ningún gastrópodo fue recolectado en estaciones más profundas que $83 \mathrm{~m}$. El número de individuos y especies varió también con el tipo de substrato. La mayor heterogeneidad de los sedimentos fue encontrada en las estaciones someras $(18-60 \mathrm{~m})$ con substratos de arena media, limo arenoso y limo arcilloso. El mayor número de especies fue recolectado en estaciones con substratos de limo arenoso y arena media. La abundancia total fue notablemente menor en arena media, mientras que la abundancia de gastrópodos vivos fue mayor en substrato de limo arcilloso. Las siete especies más abundantes representaron $51.2 \%$ de todos los individuos. La mayoría de estas especies son de interés comercial, en particular aquellas de los géneros Fusinus, Hexaplex, Ficus, Harpa, Cantharus y Bursa.

Palabras clave: gastrópodos, bentos, plataforma continental, Pacífico mexicano.

\section{INTRODUCTION}

Present knowledge of benthic mollusks of the Mexican tropical Pacific, including the coasts of Jalisco and Colima, is mainly due to studics carried out in the intertidal and shallow subtidal zones. Most investigations focus on the taxonomic composition, and the abundance and distribution patterns of gastropod communities. A recent list of these investigations and the localities studied so far is reported by Rios-Jara et al. (1996). Other investigations include the work of Hertlein and Allison (1960) on the Clipperton Islands; DuShane and Poorman (1967) in Guaymas, Sonora; Draper (1975) in Cholla Bay, Sonora; and Holguin-Quiñones et al. (1987) on several beaches of Oaxaca.

A few studies on benthic mollusk communjties have also been conducted over wider subtidal regions of the continental shelf in the states of Guerrero (Lesser-Hiriart, 1984) and Nayarit (Reguero-Reza, 1985); the southern region of Sinaloa (Hendrickx et al., 1984); the Gulf of Tehuantepec (Secretaría de Marina, 1980); and the Gulf of California (Parker, 1964; HerreraPeña, 1981; Guerrero-Pelcastre, 1986).

The malacologic benthic fauna of the continental shelf off Jalisco and Colima has not been well studied. Only a few published records are mentioned in the literature, including the collations and summations of museum collections made by Keen (1971) and Keen and Coan (1974),

\section{INTRODUCCIÓN}

El conocimiento actual sobre los moluscos bénticos del Pacífico tropical mexicano, incluyendo las costas de Jalisco y Colima, se debe principalmente a estudios llevados a cabo en las zonas intermareal y submareal somera. Estos estudios se refieren a la composición taxonómica, y a los patrones de distribución y abundancia de las comunidades de gastrópodos. Una lista reciente de estas investigaciones y las localidades hasta ahora estudiadas es presentada por Ríos-Jara et al (1996). Otras investigaciones incluyen los trabajos de Hertlein y Allison (1960) en las islas Clipperton; DuShane y Poorman (1967) en Guaymas, Sonora; Draper (1975) en Bahía Cholla, Sonora; Holguín-Quiñones et al. (1987) en varias playas de Oaxaca.

Se han realizado también algunos estudios sobre las comunidades de moluscos bénticos en regiones submareales más amplias de la plataforma continental de los estados de Guerrero (Lesser-Hiriart, 1984) y Nayarit (Reguero-Reza, 1985); el sur de Sinaloa (Hendrickx et al, 1984); el Golfo de Tehuantepec (Secretaría de Marina, 1980); y el Golfo de California (Parker, 1964; Herrera-Peña, 1981; Gucrrero-Pclcastrc, 1986).

La fauna malacológica de la plataforma continental de Jalisco y Colima no ha sido bien estudiada. Sólo pocos registros son mencionados en la literatura, los cuales incluyen los compendios y recopilaciones de colecciones de museos 
and a report of the first record of 17 species of gastropods collected in this region (Ríos-Jara et al., 1996). Therefore, the present study is important, since it will provide a more complete understanding of the taxonomic composition of the subtidal gastropod populations of this region and their spatial distribution patterns of abundance and diversity with respect to depth and type of substratum.

Collections of benthic mollusks reported here were carried out during the oceanographic expedition Atlas $V$ along the continental shelf off Jalisco and Colima, in the central portion of the tropical Mexican Pacific. A total of 2774 gastropods were collected during the expedition; 2204 individuals $(79.5 \%)$ were collected using a Van Veen grab, whereas 570 individuals $(20.5 \%)$ were obtained with a trawl net. All these gastropods belong to 242 species, 190 from the grab and 86 from the trawl net samples. This study describes the spatial distribution patterns of abundance and diversity with respect to depth and type of substratum of species collected only with the trawl net.

\section{AREA OF STUDY}

The area of study is defined by the length of the continental shelf off the states of Jalisco and Colima along the Pacific coast of Mexico. The continental shelf of both states is narrow and mostly rocky, extending across an area of $5315 \mathrm{~km}^{2}$ (Ruíz-Durán, 1985). The coast of Jalisco and Colima is characterized by its irregular and mountainous orography, with frequent cliffs, bays and heaches of diverse length and shape. This characteristic is reflected in the marine bottom, where areas of uneven topography intercalate with relatively flat zones (GuzmánArroyo and Flores-Rosas, 1988). The coastline of both states stretches along approximately $364 \mathrm{~km}$ and corresponds to a tectonic littoral type, while the northern coast of Jalisco is defined as abrupt with intense marine erosion (Galaviz-Solís and Gutiérrez-Estrada, 1978). The hydrologic system along this coastal region includes several rivers, realizados por Keen (1971) y Keen y Coan (1974), y un reporte del registro de 17 especies de gastrópodos recolectados por primera vez en esta región (Ríos-Jara et al., 1996). Esta falta de información confiere importancia al presente estudio, ya que proporcionará un mejor conocimiento de la composición taxonómica de las poblaciones de gastrópodos submareales de esta región, además de sus patrones de distribución de abundancia y diversidad con respecto a la profundidad y tipo de substrato.

Las recolecciones de moluscos bénticos reportadas en este trabajo fueron realizadas durante la expedición oceanográfica Atlas $V$ a lo largo de la plataforma continental de Jalisco y Colima, en la porción central del Pacífico tropical mexicano. Un total de 2774 gastrópodos fueron recolectados durante la expedición, 2204 individuos $(79.5 \%)$ con una draga Van Veen y 570 individuos $(20.5 \%)$ con red de arrastre. Todos estos gastrópodos pertenecen a 242 especies, de las cuales 190 fueron recolectadas con draga y 86 con red de arrastre. Este estudio describe los patrones de distribución espacial de abundancia y diversidad con respecto a la profundidad y tipo de substrato de las especies recolectadas únicamente con la red de arrastre.

\section{ÁREA DE ESTUDIO}

El área de estudio comprende la plataforma continental de los estados de Jalisco y Colima en la costa del Pacífico de México. La plataforma continental de ambos estados es angosta y mayormente rocosa, extendiéndose a través de un área de $5315 \mathrm{~km}^{2}$ (Ruiz-Durán, 1985). La costa de Jalisco y Colima se caracteriza por su orografia irregular y montañosa, con frecuentes acantilados, bahías y playas de diversa longitud y conformación. Esta característica se refleja en el fondo marino, donde están presentes zonas de topografía accidentada intercaladas con fondos relativamente planos (Guzmán-Arroyo y Flores-Rosas, 1988). La línea de costa de ambos estados se extiende aproximadamente $364 \mathrm{~km}$ y es de tipo tectónico, 
coastal lagoons and estuaries. The most important lagoons are Agua Dulce and Cuyutlán, and the principal estuaries include Maito, La Boquita, El Ermitaño, El Chorro, Loya, Majahuas, Paramán, Rodea, Porto Grande and Palo Verde (Lankford, 1977). The principal rivers of this region are, from north to south: Ameca, Pitillal, Cuate, Tecomala, Tomatlán, San Nicolás, Cuitzmala, Purificación, Cihuatlán, Río Seco, Armería and Coahuayana.

\section{MATERIALS AND METHODS}

The oceanographic expedition Atlas $V$ was carried out on board the R/V El Puma of the Universidad Nacional Autónoma de México. The expedition was divided in two phases, parts $A$ and $B$, both conducted over the continental shelf off Jalisco and Colima. Activities of part A were conducted from north to south (14-21 August, 1988 ) and activities of part B, from south to north (23-30 August, 1988).

Topographical characteristics of the area of study were determined by means of continuous registration with an echo-sounder system during part A. This information was used to locate the most favorable areas to operate a trawl net (shrimp-fishing type: $24 \mathrm{~m}$ long and $21 \mathrm{~m}$ wide, $4.5 \mathrm{~cm}$ mesh size in the wings and $0.6 \mathrm{~cm}$ at the cod end), which was used in the collection of the biological samples. Biological collections were made during part $B$ of the expedition. During this second part, 13 transects perpendicular to the coastline were established at intervals of approximately $10^{\prime}$ of latitude. The first transect (transect XIII) was located at the border of the states of Colima and Michoacán $\left(18^{\circ} 39^{\prime} \mathrm{N}\right)$, and the last transect (transect I), at the border of the states of Nayarit and Jalisco $\left(20^{\circ} 39^{\prime} \mathrm{N}\right)$ (fig. 1). One to four sampling stations were conducted in each transect. Net trawls were performed only at 22 stations (transects I, II, IV, V, VI, VII, VIII, XII and XIII). The depth of these stations ranged from 18 to $112 \mathrm{~m}$. The time of each haulage was approximately $30 \mathrm{~min}$, with a speed of 2.5 knots. All gastropod shells obtained were separated and organisms collected alive were preserved in $70 \%$ mientras que la costa norte de Jalisco se define como abrupta con erosión marina intensa (Galaviz-Solís y Gutiérrez-Estrada, 1978). El sistema hidrológico a lo largo de esta región costera incluye varios ríos, lagunas costeras y estuarios. Las lagunas más importantes son Agua Dulce y Cuyutlán, y los estuarios más importantes incluyen Maito, La Boquita, El Ermitaño, El Chorro, Loya, Majahuas, Paramán, Rodea, Porto Grande y Palo Verde (Lankford, 1977). Los ríos principales de esta región son, de norte a sur: Ameca, Pitillal, Cuate, Tecomala, Tomatlán, San Nicolás, Cuitzmala, Purificación, Río Seco, Armería y Coahuayana.

\section{MATERIALES Y MÉTODOS}

La expedición oceanográfica Atlas $V$ fue llevada a cabo a bordo del B/O El Puma de la Universidad Nacional Autónoma de México. Esta campaña estuvo dividida en dos fases, partes A y $\mathrm{B}$, ambas realizadas en la plataforma continental de los estados de Jalisco y Colima. Las actividades de la parte $A$ se realizaron de norte a sur $(14$ a 21 de agosto de 1988) y las actividades de la parte $\mathrm{B}$, de sur a norte (23 a 30 de agosto de 1988).

Las características topográficas del área de estudio fueron determinadas mediante registro continuo con una ecosonda durante la parte A. Esta información fue usada para localizar las áreas más favorables para operar una red de arrastre (tipo camaronero: $24 \mathrm{~m}$ de longitud y $21 \mathrm{~m}$ de ancho, $4.5 \mathrm{~cm}$ de luz de malla en las alas y $0.6 \mathrm{~cm}$ en el copo), la cual fue usada en la recolección de las muestras biológicas. Las recolecciones biológicas fueron realizadas durante la parte $B$ de la campaña. Durante esta segunda parte se establecieron 13 transectos perpendiculares a la línea de costa a intervalos de aproximadamente $10^{\prime}$ de latitud; el primero de ellos (transecto XIII) se localizó en el límite de los estados de Colima y Michoacán $\left(18^{\circ} 39^{\prime} \mathrm{N}\right)$ y el último (transecto I), en el límite de los estados de Nayarit y Jalisco $\left(20^{\circ} 39^{\prime} \mathrm{N}\right)$ (fig. 1). Se llevaron a cabo de una a cuatro estaciones de muestreo en cada transecto. Los arrastres se realizaron solamente en 22 


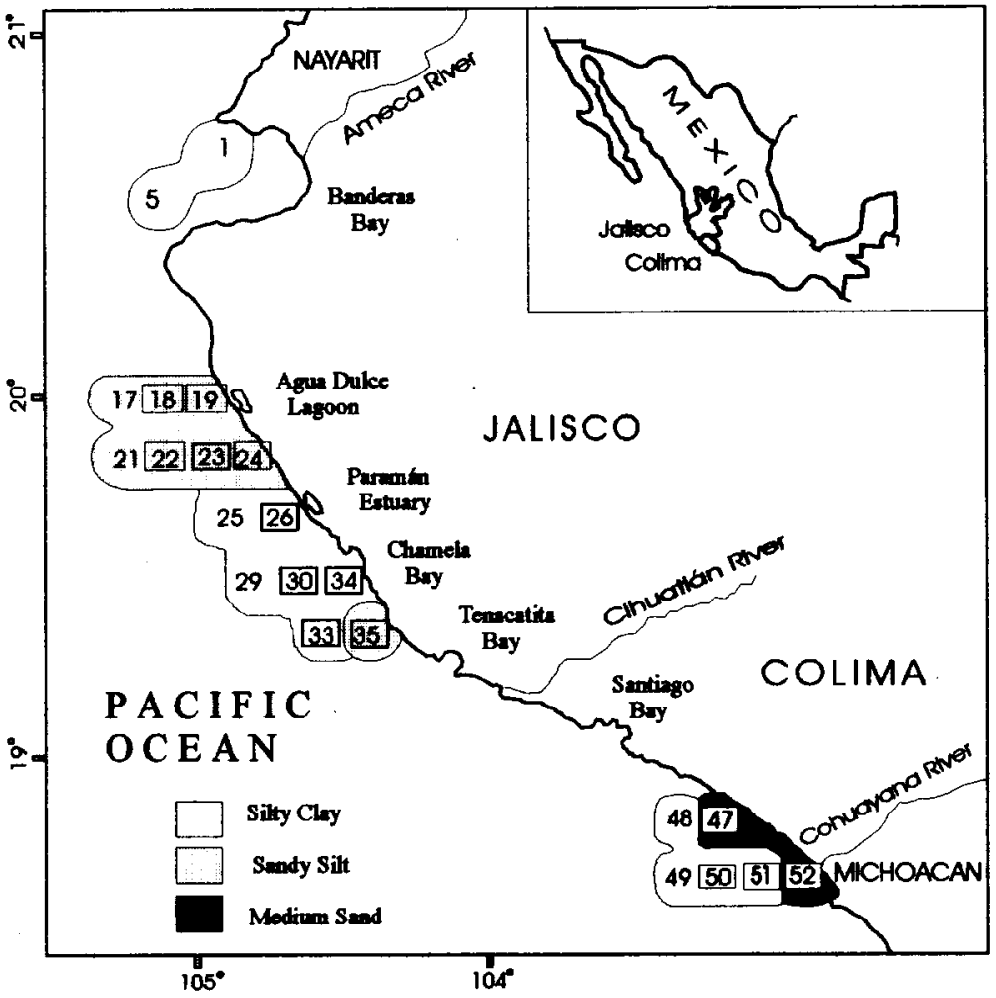

Figure 1. Location of sampling stations and distribution of types of substratum along the continental shelf off Jalisco and Colima, Mexico. Squares indicate stations with gastropods reported in this study.

Figura 1. Localización de las estaciones de muestreo y distribución de los tipos de substrato sobre la plataforma continental de Jalisco y Colima, México. Los cuadrados indican las estaciones en las que se reportan gastrópodos en este estudio.

alcohol. $\Lambda$ geological Van Veen grab $(20 \mathrm{~L}$ of capacity and $0.1 \mathrm{~m}^{2}$ of collecting surface area) was used to collect sediments in order to characterize the type of substratum of each sampling station. Samples of sediment were sieved through three different screens (mesh size $=10,3$ and $1 \mathrm{~mm}$ ) and analyzed at the Instituto de Gcografia of the Universidad de Guadalajara, Mexico

Once in the laboratory, gastropods were sorted and quantified. All individuals were identified to species. The works of Morris (1966), Keen (1971), Keen and Coan (1974) and Abbott (1974) estaciones (transectos I, II, IV, V, VI, VII, VIII, XII y XIII). La profundidad de estas estaciones varió desde 18 hasta $112 \mathrm{~m}$. El tiempo de cada arrastre fue de aproximadamente $30 \mathrm{~min}$, a una velocidad de 2.5 nudos. Todas las conchas de gastrópodos obtenidas fueron separadas y los organismos recolectados vivos fueron preservados en alcohol al $70 \%$. Se utilizó una draga geológica Van Veen $\left(20 \mathrm{~L}\right.$ de capacidad y $0.1 \mathrm{~m}^{2}$ de superficie) para recolectar sedimentos y caracterizar el tipo de substrato de cada estación de muestreo. Las muestras de sedimento fueron tamizadas a través de tres diferentes mallas (luz de malla $=10$, 
were used for identification. Only shells that permitted clear identification were used. Taxonomic names, their relationships and ranges of distribution were reviewed and updated using Skoglund's (1992) recent publication.

Samples were standardized because there were some differences in sampling intensity with respect to depths and types of substratum. Therefore, abundance was expressed as the mean number of individuals per station and diversity as the mean number of species per station, and each value referred to the average of all sampling stations within each depth group or type of substratum, respectively. Sampling stations were grouped into five depth ranges $(18-40,41-60$, 61-83, 84-112 and 113-136 m), and the abundance and diversity values of each station were used as replicates for their corresponding depth ranges.

\section{RESULTS AND DISCUSSION}

A total of 582 gastropods belonging to 3 subclasses, 4 orders, 25 families, 42 genera and 86 species were collected (table 1). The subclass Prosobranchia was the most diverse, with 23 families. Only one species, Heliacus caelatus, was registered from the subclass Heterobranchia, and only one species, Armina californica, a nonshelled gastropod (order Nudibranchia), from the subclass Opistobranchia. Eight genera represented $41.2 \%$ of all the species, with more than three species each: Conus (7), Crucibulum (5), Turritella (5), Crepidula (4), Hexaplex (4), Knefastia (5) Polystira (4) and Strombina (4). Only 281 gastropods from 33 species were collected alive. The first record in the area of study of three of these species (Knefastia howelli, $K$. walkeri and Nassarina perata) was reported by Ríos-Jara et al. (1996).

There are at least three different types of substrata in the area of study: (1) silty clay (most particles $<0.020 \mathrm{~mm})$, (2) sandy silt $(0.020$ to $0.015 \mathrm{~mm})$, and (3) medium sand $(>0.2 \mathrm{~mm})$. Most of the stations had the first two types of substratum ( 13 with silty clay and 7 with sandy silt);
3 y $1 \mathrm{~mm}$ ) y analizadas en el Instituto de Geografía de la Universidad de Guadalajara, México.

Una vez en el laboratorio, los gastrópodos fueron separados y cuantificados. Todos los individuos fueron identificados hasta especie. Los trabajos de Morris (1966), Keen (1971), Keen y Coan (1974) y Abbott (1974) fueron usados para esta identificación. Se consideraron únicamente conchas poco deterioradas que permitieran una identificación certera. Los nombres taxonómicos, sus relaciones y rangos de distribución fueron revisados y actualizados usando la reciente publicación de Skoglund (1992).

Las muestras fueron estandarizadas ya que existieron diferencias con respecto a las profundidades y tipos de substrato. La abundancia fue expresada como el número promedio de individuos por estación y la diversidad como el número promedio de especies por estación; cada valor se refiere al promedio de todas las estaciones de muestreo por cada profundidad o tipo de substrato, respectivamente. Las estaciones de muestreo fueron agrupadas en cinco intervalos de profundidad $(18-40,41-60,61-83,84-112 \mathrm{y}$ $113-136 \mathrm{~m}$ ), y los valores de abundancia y diversidad para cada estación fueron usados como réplicas para cada profundidad correspondiente.

\section{RESULTADOS Y DISCUSIÓN}

Un total de 582 gastrópodos pertenecientes a 3 subclases, 4 órdenes, 25 familias, 42 géneros y 86 especies fueron recolectados (tabla 1). La subclase Prosobranchia fue la más diversa, con 23 familias. Sólo una especie, Heliacus caelatus, fue registrada de la subclase Heterobranchia y sólo una especie, Armina californica, un gastrópodo sin concha (orden Nudibranchia), de la subclase Opistobranchia. Ocho géneros representaron el $41.2 \%$ de todas las especies, con más de tres especies cada uno: Conus (7), Crucibulum (5), Turritela (5), Crepidula (4), Hexaplex (4), Knefastia (5), Polystira (4) y Strombina (4). Únicamente 281 gastrópodos pertenecientes a 33 especies fueron recolectados vivos. El primer registro en el área de estudio de tres de estas 
Table 1. Distribution [sampling station (\#). type of substratum and mean depth (in meters)] and abundance of the gastropod species collected in the area of study. Numbers indicate individuals obtained with a trawl net at each sampling station: individuals collected alive are shown between brackets. $\mathrm{MS}=$ medium sand. $\mathrm{SS}=$ sandy silt and $\mathrm{SC}=$ silty clay.

Tabla 1. Distribución [estación de muestreo (\#). tipo de substrato y profundidad media (en metros)] y abundancia de las especies de gastrópodos recolectadas en el área de estudio. Los números indican los individuos obtenidos con la red de arrastre en cada estación de muestreo: los individuos recolectados vivos se muestran entre paréntesis. $\mathrm{MS}=$ arena media. $\mathrm{SS}=$ limo arenoso y $\mathrm{SC}=$ limo arcilloso.

\begin{tabular}{|c|c|c|c|c|c|c|c|c|c|c|c|c|c|c|c|}
\hline Species & $\begin{array}{l}\# 52 \\
\text { MS } \\
18 \mathrm{~m}\end{array}$ & $\begin{array}{l}\# 47 \\
M S \\
40 \mathrm{~m}\end{array}$ & $\begin{array}{l}1724 \\
\mathrm{SS} \\
2.3 \mathrm{~m}\end{array}$ & $\begin{array}{l}\# 19 \\
5 S \\
36 \mathrm{~m}\end{array}$ & $\begin{array}{l}\# 35 \\
S S \\
48 \mathrm{~m}\end{array}$ & $\begin{array}{l}\# 23 \\
S S \\
49 \mathrm{~m}\end{array}$ & $\begin{array}{l}\# 18 \\
\text { SS } \\
71 \mathrm{~m}\end{array}$ & $\begin{array}{l}\# 22 \\
\text { SS } \\
72 \mathrm{~m}\end{array}$ & $\begin{array}{l}\# 26 \\
S C \\
57 \mathrm{~m}\end{array}$ & $\begin{array}{l}\# 51 \\
\mathrm{SC} \\
59 \mathrm{~m}\end{array}$ & $\begin{array}{l}\# 34 \\
\mathrm{SC} \\
60 \mathrm{~m}\end{array}$ & $\begin{array}{l}\# 30 \\
\mathrm{SC} \\
73 \mathrm{~m}\end{array}$ & $\begin{array}{c}\# 33 \\
\mathrm{SC} \\
83 \mathrm{~m}\end{array}$ & $\begin{array}{l}450 \\
S C \\
83 \mathrm{~m}\end{array}$ & Total \\
\hline 1. Armina californica (Cuoper. 1862) & (1) & & & & & & & & & & & & & & 1 \\
\hline 2. Bursa nana Röding. 1798 & 1 & & & & & 10 & & $4(4)$ & $5(3)$ & (8) & 2 & & & & 37 \\
\hline 3. Calyptraea mamillaris Broderip. 1843 & (4) & & (1) & & & & & & & & & & & & 5 \\
\hline 4. Cancellaria balboae Pilsbry. 1931 & & & & & & 1 & & & & & & & & & 1 \\
\hline 5. Cancellaria obesa Sowerby. 1832 & & & & & & & & & 1 & & & & & & 1 \\
\hline $\begin{array}{l}\text { 6. Cantharus pallidus } \\
\text { (Broderip \& Sowerby. 1829) }\end{array}$ & & & & l( (1) & & 1 & (16) & 4 & 1 & & & 8 & $10(11)$ & 2 & 55 \\
\hline $\begin{array}{l}\text { 7. Compsodrillia haliplexa } \\
\text { (Dall. 1919) }\end{array}$ & & & & & & & 4 & & & & & & & & 4 \\
\hline 8. Compsodrillia sp. 1 & & & & & & 1 & & & & & & & & 1 & 2 \\
\hline 9. Conus dalli Stearns. 1873 & & & & & & & & & 1 & & & 1 & & & 2 \\
\hline 10. Conus patricius Hinds. 1843 & (2) & & & & & & & & & & & & & & 2 \\
\hline 11. Conus poormani Berry, 1968 & & & & & & 1 & & 1 & & & & & & & 2 \\
\hline 12. Conus princeps Linnaeus. 1758 & & & & & (2) & & & (1) & 1 & & & & & & 4 \\
\hline $\begin{array}{l}\text { 13. Comus purpurascens } \\
\text { Sowerby: } 1833\end{array}$ & & & & & & & & 1 & & & & & & & 1 \\
\hline 14. Conus regularis Sowerby, 1833 & & & & & 1 & 2 & & $3(1)$ & 6 & & & & & & 13 \\
\hline 15. Conus tornatus Sowerby, 1833 & & & & & & & & 2 & & & & & & & 2 \\
\hline
\end{tabular}


Table 1 (Cont.)

\begin{tabular}{|c|c|c|c|c|c|c|c|c|c|c|c|c|c|c|c|}
\hline Species & $\begin{array}{c}\# 52 \\
\mathrm{MS} \\
18 \mathrm{~m}\end{array}$ & $\begin{array}{c}\# 47 \\
\mathrm{MS} \\
40 \mathrm{~m}\end{array}$ & $\begin{array}{c}\# 24 \\
\mathrm{SS} \\
23 \mathrm{~m}\end{array}$ & $\begin{array}{c}\# 19 \\
\text { SS } \\
36 \mathrm{~m}\end{array}$ & $\begin{array}{c}\# 35 \\
S S \\
48 \mathrm{~m}\end{array}$ & $\begin{array}{c}\# 23 \\
\mathrm{SS} \\
49 \mathrm{~m}\end{array}$ & $\begin{array}{c}\# 18 \\
\mathrm{SS} \\
71 \mathrm{~m}\end{array}$ & $\begin{array}{c}\# 22 \\
\text { SS } \\
72 \mathrm{~m}\end{array}$ & $\begin{array}{c}\# 26 \\
\mathrm{SC} \\
57 \mathrm{~m}\end{array}$ & $\begin{array}{c}\# 51 \\
\mathrm{SC} \\
59 \mathrm{~m}\end{array}$ & $\begin{array}{c}\# 34 \\
\mathrm{SC} \\
60 \mathrm{~m}\end{array}$ & $\begin{array}{c}\# 30 \\
\mathrm{SC} \\
73 \mathrm{~m}\end{array}$ & $\begin{array}{c}\# 33 \\
\mathrm{SC} \\
83 \mathrm{~m}\end{array}$ & $\begin{array}{c}\# 50 \\
\mathrm{SC} \\
83 \mathrm{~m}\end{array}$ & Total \\
\hline 16. Coralliophila orcuttiana Dall, 1919 & & & & & & & 2 & & & & & & & & 2 \\
\hline $\begin{array}{l}\text { 17. Costoanachis nigricans } \\
\text { (Sowerby, 1844) }\end{array}$ & & & 4 & . & & & & & & & & & & & 4 \\
\hline $\begin{array}{l}\text { 18. Crassispira ballenaensis } \\
\text { Hertlein \& Strong. } 1951\end{array}$ & & & & & & 1 & & & & & & & & & 1 \\
\hline 19. Crassispira kluthi E.K. Jordan, 1936 & & & & & & 1 & & & & & & & & & 1 \\
\hline 20. Crassispira tepocana (Dall. 1919) & & & & 1 & & & & & & & & & & & 1 \\
\hline 21. Crepidula aculeata (Gmelin, 1791) & & (1) & $7(4)$ & & (2) & & & & & & & & & & 14 \\
\hline 22. Crepidula excavata (Broderip, 1834) & (4) & & $1(12)$ & & & & & & & & & & & & 17 \\
\hline 23. Crepidula onyx Sowerby, 1824 & & & $2(4)$ & (4) & (6) & & & & 1 & & & & & & 17 \\
\hline $\begin{array}{l}\text { 24. Crepidula perforans } \\
\text { (Valenciennes, 1846) }\end{array}$ & (2) & (1) & (24) & & & 1 & & & (1) & & & & & & 29 \\
\hline $\begin{array}{l}\text { 25. Crucibulum lignarium } \\
\text { (Broderip, 1834) }\end{array}$ & & & & (3) & $1(2)$ & & & (1) & (2) & & & (1) & (2) & & 12 \\
\hline 26. Crucibulum monticulus Berry, 1969 & & (1) & & & & & & & & & & & & & 1 \\
\hline 27. Crucibulum scutellatum (Wood, 1928) & & 1 & (1) & (1) & & 1 & & & & & & & & & 4 \\
\hline 28. Crucibulum spinosum (Sowerby, 1824) & & & $2(8)$ & & (2) & & & & & & & & & & 12 \\
\hline 29. Crucibulum umbrella (Deshayes, 1830) & & & (1) & & & & & & & & & & & & 1 \\
\hline 30. Distorsio constricta (Broderip. 1833) & & & 1 & & & & & & & & & & & & 1 \\
\hline $\begin{array}{l}\text { 31. Distorsio decussata } \\
\text { (Valenciennes. 1832) }\end{array}$ & & & & & & & & 1 & 2 & & & & & & 3 \\
\hline $\begin{array}{l}\text { 32. Drillia valida } \\
\text { McLean \& Poorman. } 1971\end{array}$ & & & & & & & 1 & & & & & & & & l \\
\hline
\end{tabular}


Table 1 (Cont.)

\begin{tabular}{|c|c|c|c|c|c|c|c|c|c|c|c|c|c|c|c|}
\hline Species & $\begin{array}{c}\# 52 \\
\text { MS } \\
18 \mathrm{~m}\end{array}$ & $\begin{array}{c}\# 47 \\
\text { MS } \\
40 \mathrm{~m}\end{array}$ & $\begin{array}{l}\# 24 \\
\text { SS } \\
23 \mathrm{~m}\end{array}$ & $\begin{array}{l}\# 19 \\
\text { SS } \\
36 \mathrm{~m}\end{array}$ & $\begin{array}{l}\# 35 \\
\text { SS } \\
48 \mathrm{~m}\end{array}$ & $\begin{array}{c}\# 23 \\
\text { SS } \\
49 \mathrm{~m}\end{array}$ & $\begin{array}{l}\# 18 \\
5 S \\
71 \mathrm{~m}\end{array}$ & $\begin{array}{l}\# 22 \\
\text { SS } \\
72 \mathrm{~m}\end{array}$ & $\begin{array}{l}\# 26 \\
S C \\
57 \mathrm{~m}\end{array}$ & $\begin{array}{c}\# 51 \\
S C \\
59 \mathrm{~m}\end{array}$ & $\begin{array}{c}\# 34 \\
\mathrm{SC} \\
60 \mathrm{~m}\end{array}$ & $\begin{array}{l}\# 30 \\
\mathrm{SC} \\
73 \mathrm{~m}\end{array}$ & $\begin{array}{c}\# 33 \\
\mathrm{SC} \\
83 \mathrm{~m}\end{array}$ & $\begin{array}{l}\mathrm{H} 50 \\
\mathrm{SC} \\
83 \mathrm{~m}\end{array}$ & Total \\
\hline 33. Enaeta barnesii (Gray, 1825) & & & & & & & & & 1 & & & & & & 1 \\
\hline 34. Ficus ventricosa (Sowerby, 1825) & & 2 & 2 & $2(1)$ & $1(4)$ & & $4(5)$ & $1(5)$ & $6(6)$ & & & 9 & 1 & & 49 \\
\hline 35. Fusinus dupetitthouarsi (Kiener, 1846) & & & & & & 6 & (9) & $3(25)$ & 1 & & (1) & $4(1)$ & & & 50 \\
\hline 36. Harpa conoidalis Röding, 1798 & & $1(1)$ & & $2(3)$ & (2) & 5 & & & $3(12)$ & (1) & & 1 & & & 31 \\
\hline $\begin{array}{l}\text { 37. Haustellum recurvirrostris } \\
\text { (Broderip, 1833) }\end{array}$ & & & & & & 3 & & 1 & & & & & & & 4 \\
\hline 38. Heliacus caelatus (Hinds. 1844) & & & & & & & & 1 & & & & & & & 1 \\
\hline 39. Hexaplex ambiguus (Reeve, 1845) & & & (3) & & & & & & & & & & & & 3 \\
\hline 40. Hexaplex brassica (Lamarck, 1822) & & 1 & 1 & (2) & (1) & & & $5(24)$ & $3(7)$ & & & 2 & 2 & & 48 \\
\hline 41. Hexaplex radix (Gmelin. 1791) & & & (1) & & & & & & & & & & & & l \\
\hline 42. Hexaplex regius (Swainson. 1821) & & & $5(3)$ & & & & & & (1) & & & & & & 9 \\
\hline 43. Hipponix grayanus Menke, 1853 & & (3) & & & & & & & & & & & & & 3 \\
\hline 44. Hipponix planatus Carpenter, 1857 & & (1) & & & & & & & & & & & & & 1 \\
\hline 45. Knefastia funiculata (Kiener, 1840) & & & & 1 & & & & & & & & & & & 1 \\
\hline $\begin{array}{l}\text { 46. Knefastia howelli (Hertlein \& Strong. } \\
\text { 1951) }\end{array}$ & & & & & & 1 & & & & & & & & & 1 \\
\hline 47. Knefastia olivacea (Sowerby, 1833) & & & & & & 3 & & & & & & & & & 3 \\
\hline 48. Knefastia walkeri Berry, 1958 & & & & & & 23 & & & & & & & & & 23 \\
\hline 49. Knefastia sp. 1 & & & & 5 & & & & & & & & & & & 5 \\
\hline 50. Latirus concentricus (Reeve. 1847) & & & & & & & & & 1 & & & & & & 1 \\
\hline 51. Latirus praestantior Melvill, 1892 & & & & & & & & & 1 & & & & & & 1 \\
\hline 52. Linatella wiegmanni (Anton, 1839) & & & & & & & & 1 & & & & & & & 1 \\
\hline
\end{tabular}


Table 1 (Cont.)

\begin{tabular}{|c|c|c|c|c|c|c|c|c|c|c|c|c|c|c|c|}
\hline Species & $\begin{array}{c}\# 52 \\
M S \\
18 \mathrm{~m}\end{array}$ & $\begin{array}{c}\# 47 \\
\mathrm{MS} \\
40 \mathrm{~m}\end{array}$ & $\begin{array}{c}\# 24 \\
\text { ss } \\
23 \mathrm{~m}\end{array}$ & $\begin{array}{c}\# 19 \\
\text { Ss } \\
36 \mathrm{~m}\end{array}$ & $\begin{array}{c}\# 35 \\
\mathrm{SS} \\
48 \mathrm{~m}\end{array}$ & $\begin{array}{c}\# 23 \\
\text { Ss } \\
49 \mathrm{~m}\end{array}$ & $\begin{array}{c}\# 18 \\
\text { SS } \\
71 \mathrm{~m}\end{array}$ & $\begin{array}{c}\# 22 \\
S \mathrm{~S} \\
72 \mathrm{~m}\end{array}$ & $\begin{array}{c}\# 26 \\
\mathrm{SC} \\
57 \mathrm{~m}\end{array}$ & $\begin{array}{c}\# 51 \\
\mathrm{SC} \\
59 \mathrm{~m}\end{array}$ & $\begin{array}{c}\# 34 \\
\mathrm{SC} \\
60 \mathrm{~m}\end{array}$ & $\begin{array}{c}\# 30 \\
\mathrm{SC} \\
73 \mathrm{~m}\end{array}$ & $\begin{array}{c}\# 33 \\
S C \\
83 \mathrm{~m}\end{array}$ & $\begin{array}{c}\# 50 \\
\mathrm{SC} \\
83 \mathrm{~m}\end{array}$ & Total \\
\hline 53. Malea ringens (Swainson, 1822) & & & 1 & & & & & & & & & & & & 1 \\
\hline 54. Malea sp. 1 & & & & & & & & 1 & & & & 1 & & & 2 \\
\hline 55. Mitra belcheri Hinds, 1843 & & & & & & & & & (2) & & & & & & 2 \\
\hline $\begin{array}{l}\text { 56. Mitra swainsonii swainsonii } \\
\text { Broderip. } 1836\end{array}$ & & & & & & & & 1 & & & & & & & 1 \\
\hline 57. Nassarina perata Keen. 1971 & & & & & & 1 & & & & & & & & & 1 \\
\hline $\begin{array}{l}\text { 58. Northia pristis } \\
\text { (Deshayes in Lamarck. 1844) }\end{array}$ & 1 & & & & & & & & & & & & & & 1 \\
\hline 59. Oliva incrassata Lightfoot. 1786 & & & 1 & & & & & & & & & & & & 1 \\
\hline 60. Oliva polpasta Duclos, 1935 & & & & & (1) & 4 & 1 & 3 & 6 & & & & & & 15 \\
\hline 61. Oliva splendidula Sowerby. 1825 & & & & & & & & & 1 & & & & & & 1 \\
\hline $\begin{array}{l}\text { 62. Pleuroploca princeps } \\
\text { (Sowerby: 1825) }\end{array}$ & & & & (1) & (2) & (1) & & & $1(1)$ & & & & & & 6 \\
\hline 63. Pleuroploca salmo (Wood 1828) & & & & & & (1) & & & & & & & & & 1 \\
\hline 64. Polinices helicoides (Gray. 1825) & & & & & & & 1 & & & & & & & & 1 \\
\hline 65. Polystira nobilis (Hinds. 1843) & & & & & & & & $1(1)$ & & & & 2 & & & 4 \\
\hline 66. Polystira oxytropis (Sowerby. 1834) & & & & & & 4 & 1 & & & & & & & & 5 \\
\hline 67. Polystira picta (Reeve, 1843) & & & & & & 2 & & & 1 & & & & & & 3 \\
\hline 68. Polystirasp. 1 & & & & 1 & & & & & & & & & & & 1 \\
\hline 69. Simum grayi (Deshayes. 1843) & & & & & & & & & 1 & & & & & & 1 \\
\hline 70. Strombina angularis (Sowerby. 1832) & & & & 1 & & & & & & & & & & & 1 \\
\hline 71. Strombina fusinoidea Dall. 1916 & & & & & & & 1 & & & & & & & & 1 \\
\hline
\end{tabular}


Table 1 (Cont.)

\begin{tabular}{|c|c|c|c|c|c|c|c|c|c|c|c|c|c|c|c|}
\hline Species & $\begin{array}{l}\# 52 \\
\text { MS } \\
18 \mathrm{~m}\end{array}$ & $\begin{array}{c}\# 47 \\
\text { MS } \\
40 \mathrm{~m}\end{array}$ & $\begin{array}{c}\# 24 \\
\text { SS } \\
23 \mathrm{~m}\end{array}$ & $\begin{array}{c}\# 19 \\
\text { SS } \\
36 \mathrm{~m}\end{array}$ & $\begin{array}{c}\# 35 \\
\text { SS } \\
48 \mathrm{~m}\end{array}$ & $\begin{array}{c}\# 23 \\
\text { SS } \\
49 \mathrm{~m}\end{array}$ & $\begin{array}{c}\# 18 \\
\text { SS } \\
71 \mathrm{~m}\end{array}$ & $\begin{array}{c}\# 22 \\
\text { SS } \\
72 \mathrm{~m}\end{array}$ & $\begin{array}{c}\# 26 \\
\mathrm{SC} \\
57 \mathrm{~m}\end{array}$ & $\begin{array}{c}\# 51 \\
\mathrm{SC} \\
59 \mathrm{~m}\end{array}$ & $\begin{array}{c}\# 34 \\
\mathrm{SC} \\
60 \mathrm{~m}\end{array}$ & $\begin{array}{c}\# 30 \\
\mathrm{SC} \\
73 \mathrm{~m}\end{array}$ & $\begin{array}{c}\# 33 \\
\text { SC } \\
83 \mathrm{~m}\end{array}$ & $\begin{array}{c}\# 50 \\
\mathrm{SC} \\
83 \mathrm{~m}\end{array}$ & Total \\
\hline 72. Strombina pavonina (Hinds, 1814) & & & & & & & & & 1 & & & & & & 1 \\
\hline $\begin{array}{l}\text { 73. Strombina pulcherrima } \\
\text { (Sowerby 1832) }\end{array}$ & & & & & & 1 & & & & & & & & & 1 \\
\hline 74. Terebra intertincta Hinds, 1844 & & & & & & & 1 & & & & & & & & 1 \\
\hline 75. Terebra larvaeformis Hinds, 1844 & & & & 2 & & & & & & & & & & & 2 \\
\hline 76. Terebra lucana Dall. 1908 & & & & & & & & & 1 & & & & & & 1 \\
\hline 77. Tiarrituris libya (Dall. 1919) & & 1 & & & & 1 & . & & & & & (1) & & & 3 \\
\hline 78. Tiarrituris spectabilis Berry, 1958 & & & & 2 & & 2 & & (2) & 1 & & & & & & 7 \\
\hline 79. Trajana perideris (Dall, 1910) & & & & & 1 & & 11 & & & & & & & & 12 \\
\hline 80. Turbo squamiger Reeve, 1843 & & & & & & & & & 1 & & & & & & 1 \\
\hline $\begin{array}{l}\text { 81. Turritella clarionensis } \\
\text { Hertlein \& Strong, } 1951\end{array}$ & & & & & & & & & 1 & & & & & & 1 \\
\hline 82. Turritella mariana Dall, 1908 & & & 1 & & & 4 & & 1 & 1 & & & & & & 7 \\
\hline 83. Turritella rubescens Reeve, 1849 & & & & & & 1 & & & & & & & & & 1 \\
\hline 84. Turritella willetti McLean, 1970 & & & & & & 2 & & & & & & & & & 2 \\
\hline 85. Turritella sp. 1 & & & & & & & & & 1 & & & 1 & & & 2 \\
\hline 86. Vasum caestus (Broderip. 1833) & & & (2) & & & & & & & & & & & & 2 \\
\hline Total individuals per station & 15 & 14 & 92 & 34 & 28 & 85 & 57 & 99 & 85 & 9 & 3 & 32 & 26 & 3 & 582 \\
\hline Live individuals per station & 13 & 8 & 64 & 16 & 24 & 2 & 30 & 64 & 35 & 9 & 1 & 3 & 12 & 0 & 281 \\
\hline Total species per station & 7 & 10 & 19 & 15 & 12 & 28 & 12 & 21 & .31 & 2 & 2 & 11 & 4 & 2 & 86 \\
\hline I ive species per station & 5 & 6 & 12 & 8 & 10 & 2 & 3 & 9 & 9 & 2 & 1 & 3 & 2 & 0 & 33 \\
\hline
\end{tabular}


only two stations had medium sand substratum. Greater heterogeneity of the sediments was registered at sampling stations located in shallower parts (18-60 m) (fig. 2). Sediments became more homogeneous closer to the deeper stations, decreasing in particle size, from medium sand and sandy silt to silty clay.

Since most of the gastropods collected were no longer alive (shells without soft parts), considerations on their distribution and abundance should be taken with caution. Although eight trawls were performed at deeper stations (84-112 m; sampling stations $1,5,17,21,25,29$, 48 and 49) (table 1, fig. 1), no additional gastropods were taken at those stations. Therefore, the number of stations was reduced from 22 to 14 .

Figure 3 shows the abundance of individuals and number of species with respect to depth and type of substratum. The total number of individuals and the total number of species refer to the mean number of all individuals and species (dead + live organisms) per sampling station within each depth group and type of substratum, respectively. Abundance of individuals was similar between 18 and $83 \mathrm{~m}$. However, the mean number of live gastropods collected per station decreased between 41 and $60 \mathrm{~m}$. The number of species found between 18 and $60 \mathrm{~m}$ was very similar. Deep $(61-83 \mathrm{~m})$ stations registered the lowest number of total species. Most species collected between 18 and $40 \mathrm{~m}$ were live organisms. However, the mean number of species collected alive per station decreased with depth. The greatest mean number of species per station was collected from sandy silt ( 11.0 total, 4.3 alive) and medium sand ( 8.0 total, 5.0 alive). In silty clay, mean numbers were smaller ( 5.8 total, 2.0 alive). The distribution of abundance with respect to the type of substratum indicates that higher mean numbers of the total and live individuals per station were found in sandy silt (35.0) and silty clay (36.2). Mean numbers of total individuals collected in medium sand substrata were notably smaller (14.5). Abundance of live gastropods was similar in medium sand and sandy silt (10.5 and 11.8, respectively). The number of live individuals was higher in silty clay (18.2). especies (Knefastia howelli, $K$. walkeri y Nassarina perata) fue reportado por Ríos-Jara et al. (1996).

Existen al menos tres diferentes tipos de substrato en el área de estudio: (1) limo arcilloso (la mayoría de las partículas $<0.020 \mathrm{~mm}$ ), (2) limo arenoso $(0.020-0.015 \mathrm{~mm})$ y (3) arena media $(>0.2 \mathrm{~mm})$. La mayoría de las estaciones registraron los dos primeros tipos de substrato (13 con limo arcilloso y 7 con limo arenoso); solamente dos estaciones registraron arena media. La mayor heterogeneidad de los sedimentos fue registrada en las estaciones de muestreo localizadas en áreas someras $(18-60 \mathrm{~m})$ (fig. 2). Los sedimentos fueron cada vez más homogéneos en las estaciones más profundas, disminuyendo su tamaño de partícula, desde arena media y limo arenoso hasta limo arcilloso.

Ya que la mayor parte de los gastrópodos se encontraron muertos (únicamente conchas sin partes blandas), las consideraciones sobre la distribución y abundancia de estos organismos deben tomarse con reserva. Aunque se realizaron ocho arrastres en estaciones más profundas $(84-112 \mathrm{~m}$; estaciones de muestreo $1,5,17,21,25,29,48$ y 49) (tabla 1, fig. 1), no se registraron gastrópodos en esas estaciones. Por tanto, el número de estaciones se redujo de 22 a 14 .

La figura 3 muestra la abundancia de gastrópodos recolectados y el número de especies con respecto a la profundidad y tipo de substrato. El número total de individuos y el número total de especies se refieren al número promedio de todos los individuos (organismos muertos + vivos) y especies por estación de muestreo dentro de cada grupo de profundidad y tipo de substrato, respectivamente. La abundancia de individuos fue similar entre los 18 y $83 \mathrm{~m}$. Sin embargo, el número promedio de gastrópodos vivos recolectados por estación disminuyó entre los 41 y $60 \mathrm{~m}$. El número de especies encontradas entre los $18 \mathrm{y}$ $60 \mathrm{~m}$ de profundidad fue muy similar. Las estaciones profundas $(61-83 \mathrm{~m})$ registraron el número promedio más bajo de especies totales. La mayoría de las especies recolectadas entre los 18 y $40 \mathrm{~m}$ fueron organismos vivos. Sin embargo, el número promedio de especies recolectadas vivas 


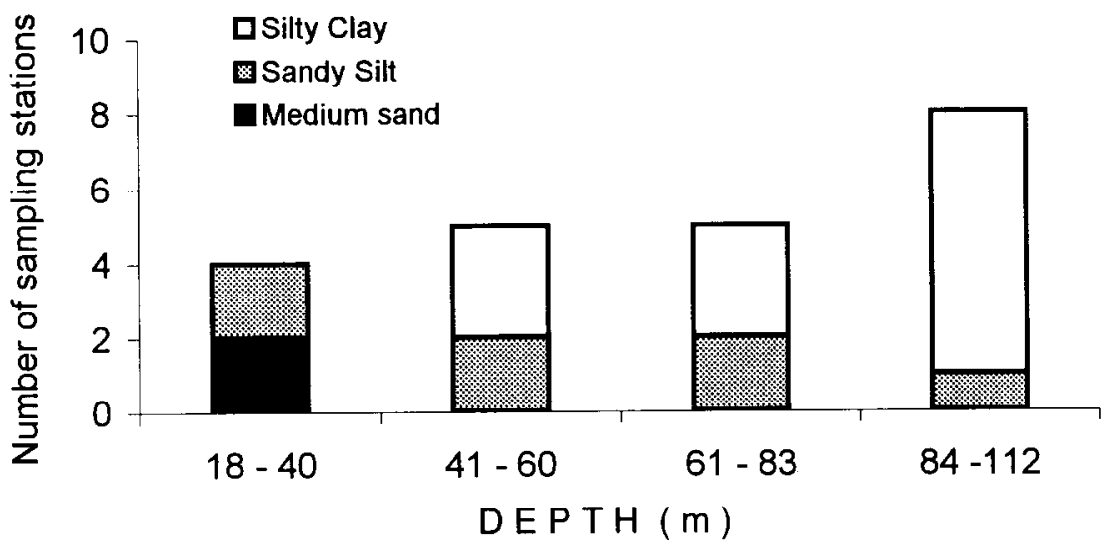

Figure 2. Distribution of the different types of substratum in relation to depth and sampling stations. Figura 2. Distribución de los diferentes tipos de substrato en relación con la profundidad y las estaciones de muestreo.

Seven species were the most abundant in the samples, representing $51.2 \%$ of the total of individuals: Cantharus pallidus (9.4\%), Fusinus dupetitthouarsi $(8.5 \%)$, Ficus ventricosa $(8.4 \%)$, Hexaplex brassica (8.3\%), Harpa conoidalis $(5.3 \%)$, Bursa nana $(6.3 \%)$ and Crepidula perforans $(5.0 \%$ ) (table 2 ). These species were collected at depths between 18 and $83 \mathrm{~m}$. A greater abundance was registered between 65 and $83 \mathrm{~m}$, with the exception of $H$. conoidalis and $B$. nana $(41-64 \mathrm{~m})$ and $C$. perforans $(18-40 \mathrm{~m})$ (table 1). Abundance was also greater in sandy silt and silty clay substrata. Only a few organisms were collected in medium sand.

The great abundance and variety of species found during this study indicate the importance of gastropods in the benthic communities of the continental shelf off Jalisco and Colima. In all biological samples obtained during the trawling activities, the malacofauna and, in particular, gastropods represented an important component of the catches, together with fish and crustaceans. por estación disminuyó con la profundidad. El número promedio mayor de especies por estación fue recolectado en limo arenoso (11.0 total, 4.3 vivas) y arena media ( 8.0 total, 5.0 vivas). En limo arcilloso los números promedio fueron menores (5.8 total, 2.0 vivas). La distribución de la abundancia con respecto al tipo de substrato indica que los números promedio mayores de los individuos totales y vivos por estación fueron encontrados en limo arenoso (35.0) y limo arciIloso (36.2). Los números promedio de individuos totales recolectados en arena media fueron notablemente menores (14.5). La abundancia de gastrópodos vivos fue similar en arena media y limo arenoso (10.5 y 11.8 , respectivamente). El número de individuos vivos fue mayor en limo arcilloso (18.2).

Siete especies fueron las más abundantes en las muestras, representando $51.2 \%$ del total de individuos: Cantharus pallidus $(9.4 \%)$, Fusinus dupetitthouarsi $(8.5 \%)$, Ficus ventricosa $(8.4 \%)$, Hexaplex brassica (8.3\%), Harpa conoidalis 

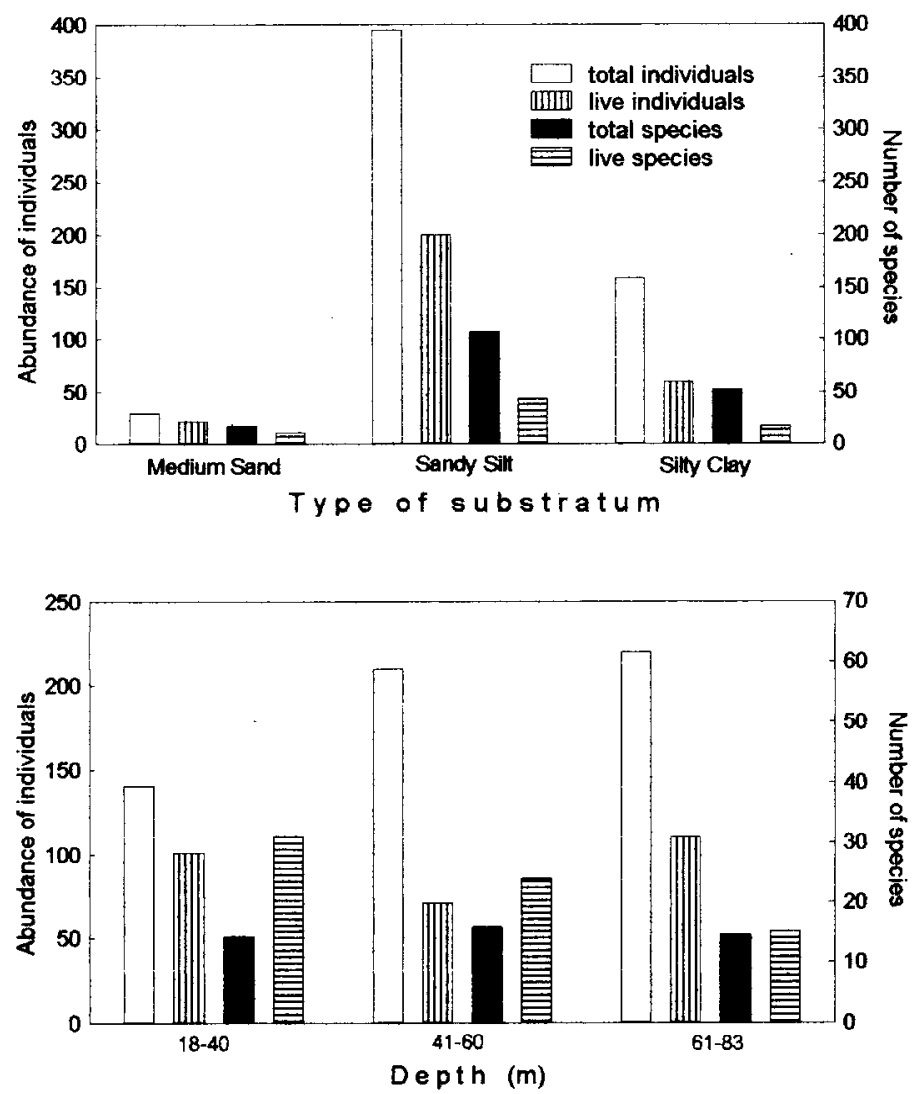

Figure 3. Abundance of individuals and number of species with respect to depth and type of substratum. Total individuals and total species refer to the mean number of all individuals and species (dead + live organisms) per sampling station within each depth group and type of substratum, respectively.

Figura 3. Abundancia de individuos y número de especies con respecto a la profundidad y el tipo de substrato. El número total de individuos y de especies se refiere al número promedio de todos los individuos y de todas las especies (organismos muertos + vivos) por estación de muestreo dentro de cada grupo de profundidad y tipo de substrato, respectivamente.

The increase in homogeneity of sediments towards deeper stations probably indicates the transient nature of subtidal environments along the continental shelf. Researchers of the benthic communities of the continental shelf (Sanders, 1968; Wildish, 1977; Warwick and Uncles, 1980; Ambrogi et al., 1990; Pérez-Peña, 1994) suggest that water depth and the type of sediment strongly influence both species richness and temporal
(5.3\%), Bursa nana $(6.3 \%)$ y Crepidula perforans $(5.0 \%)$ (tabla 2). Estas especies fueron recolectadas a profundidades de entre 18 y $83 \mathrm{~m}$. Una mayor abundancia fue registrada entre $\operatorname{los} 65 \mathrm{y}$ $83 \mathrm{~m}$, con la excepción de $H$. conoidalis y $B$. nana (41-64 m) y C. perforans (18-40 m) (tabla 1). La abundancia fue también mayor en substratos limo arenoso y limo arcilloso. Pocos organismos fueron recolectados en arena media. 
Table 2. Abundance of the most representative gastropod species in the area of study. MS $=$ medium sand, $\mathrm{SS}=$ sandy silt and $\mathrm{SC}=$ silty clay.

Tabla 2. Abundancia de las especies de gastrópodos más representativas en el área de estudio. MS = arena media, $\mathrm{SS}=$ limo arenoso y $\mathrm{SC}=$ limo arcilloso.

\begin{tabular}{lccccc}
\hline Species & $\begin{array}{c}\text { Abundance } \\
\text { (individuals } \\
\text { per trawl) }\end{array}$ & $\begin{array}{c}\text { Relative } \\
\text { abundance } \\
(\%)\end{array}$ & $\begin{array}{c}\text { Cumulative } \\
\text { relative } \\
\text { abundance } \\
(\%)\end{array}$ & $\begin{array}{c}\text { Type of } \\
\text { substratum }\end{array}$ & $\begin{array}{c}\text { Depth } \\
\text { range } \\
(\mathrm{m})\end{array}$ \\
\hline 1. Canthurus pallidus & 55 & 9.4 & 9.4 & SS, SC & $36-83$ \\
2. Fusinus dupetittouarsi & 50 & 8.5 & 17.9 & SS, SC & $49-73$ \\
3. Ficus ventricosa & 49 & 8.4 & 26.3 & SS, SC, MS & $23-83$ \\
4. Hexaplex brassica & 48 & 8.3 & 34.6 & SS, SC, MS & $23-83$ \\
5. Bursa nana & 37 & 6.3 & 40.9 & SS, SC & $18-72$ \\
6. Harpa conoidalis & 31 & 5.3 & 46.2 & SS, SC, MS & $36-73$ \\
7. Crepidula perforans & 29 & 5.0 & 51.2 & SS, SC, MS & $18-57$ \\
\hline
\end{tabular}

stability of the resident biota. Sediment composition of the marine bottom in the area of study presents greater substratum heterogeneity in the shallower regions near the coast. Gastropods collected at stations with depths less than $60 \mathrm{~m}$ were distributed in at least three different types of substratum and registered higher diversity than deeper stations. A greater heterogeneity in particle size of the sediments and texture of the sea bottom is probably related to a greater variety of benthic habitats and, therefore, a greater variety of species.

Sanders and Hessler (1969) suggest that depth rather than distance from land determine the food supply for the benthic fauna in the deeper regions of the continental shelf because decomposition removes most utilizable energy during the sinking of particles. Thus, a lower food supply must account for the reduced biomass with increasing depth. Changes in the faunal biomass below 100 to $300 \mathrm{~m}$ depth do generally correspond to the vertical distribution of other variables, which decrease rapidly with increasing depth (Kiorbe,
La gran abundancia y variedad de especies encontradas durante el presente estudio indican la importancia de los gastrópodos en las comunidades bénticas de la plataforma continental de Jalisco y Colima. En todas las muestras biológicas obtenidas durante los arrastres, la malacofauna y, particularmente, los gastrópodos representaron un componente importante de las capturas, junto con los peces y los crustáceos.

$\mathrm{El}$ incremento en homogeneidad de los sedimentos hacia las estaciones más profundas probablemente indica la naturaleza transicional de los ambientes submareales a través de la plataforma continental. Investigadores de comunidades bénticas de la plataforma continental (Sanders, 1968; Wildish, 1977; Warwick y Uncles, 1980; PérezPeña, 1994) sugieren que la profundidad y el tipo de sedimento influyen fuertemente sobre la riqueza y estabilidad temporal de la biota residente. La composición del sedimento en el área de estudio presenta mayor heterogeneidad en las regiones someras cerca de la costa. Los gastrópodos recolectados en estaciones con profundidades 
1979; Parsons et al., 1984), so several factors may be involved.

Gastropods were found as deep as $83 \mathrm{~m}$ on the continental shelf during the present study. The absence of organisms at deeper stations is probably related to a marked decrease in dissolved oxygen concentrations $\left(<0.8 \mathrm{mg} \mathrm{L}^{-1}\right)$, recorded in waters deeper than $100 \mathrm{~m}$ of this region (GuzmanArroyo and Flores-Rosas, 1988). The impingement of a minimum oxygen layer onto a continental shelf or low oxygen levels in the deeper regions of the shelf with restricted circulation may limit total biomass and species composition of the benthic communities (Parsons et al., 1984; Frigiolos and Zenetos, 1988). Low dissolved oxygen concentrations have been mentioned as the principal limiting factor for the distribution of benthic mollusks in the Gulf of California (Guerrero-Pelcastre, 1986) and the continental shelf off Guerrero, Mexico (Lesser-Hiriart, 1984).

Seven of the most abundant species in the samples are epifaunal carnivores, except $C$. perforans (Lindner, 1975). Epifaunal species tend to have a greater variety of size and feeding habits than infaunal species (Eagle, 1975; Buchanan et al., 1978), indicating a greater adaptive radiation. Most of the individuals of these species were captured alive in the three types of substratum found in the area of study. They constitute an important component of the fauna accompanying commercial shrimp fisheries in the Mexican Pacific (García-Cubas et al., 1986). These species are of considerable size and they are used as food, bait and in local craftsmanship (Cifuentes-Lemus, 1986).

\section{ACKNOWLEDGEMENTS}

This research was conducted with the aid of field and lab equipment of the Universidad de Guadalajara (UG) and the Universidad Nacional Autónoma de México. All persons in the Laboratorio de Ecologia Marina of the Facultad de Ciencias (UG) offered us much help during the field and laboratory work, especially Ernesto López-Uriarte, Lucía Lizárraga and Samuel menores que $60 \mathrm{~m}$ estuvieron distribuidos en los tres tipos de substrato y registraron mayor diversidad que las estaciones más profundas. La mayor heterogeneidad del tamaño de partícula de los sedimentos y textura del fondo marino está probablemente relacionada con una mayor variedad de hábitats bénticos $\mathrm{y}$, por lo tanto, con una mayor variedad de especies.

Sanders y Hessler (1969) sugieren que es más bien la profundidad y no la distancia de la costa la que determina el abastecimiento de alimento para la fauna béntica en las regiones más profundas de la plataforma continental debido a que la descomposición elimina la mayor parte de la energía utilizable durante el hundimiento lento de las partículas. De esta manera, el menor abastecimiento de alimento causa una reducción en la biomasa al aumentar la profundidad. Los cambios en la biomasa de la fauna debajo de los 100 a $300 \mathrm{~m}$ de profundidad generalmente corresponden a la distribución vertical de otras variables, las cuales disminuyen rápidamente al aumentar la profundidad (Parsons et al., 1984), por lo que varios factores pueden estar involucrados.

Algunos gastrópodos recolectados vivos fueron encontrados a $83 \mathrm{~m}$ en la plataforma continental durante el presente estudio. La ausencia de organismos vivos en estaciones más profundas está probablemente relacionada con un marcado descenso en las concentraciones de oxígeno disuelto $\left(<0.8 \mathrm{mg} \mathrm{L}^{-1}\right)$, el cual fue registrado en aguas de profundidades mayores que $100 \mathrm{~m}$ en esta región (Guzmán-Arroyo y Flores-Rosas, 1988). La intrusión de una capa de concentraciones mínimas de oxígeno a la plataforma continental o la aparición de niveles bajos de oxígeno en las regiones más profundas de la plataforma continental con circulación restringida, pueden limitar la biomasa total y la composición de especies de las comunidades bénticas (Parsons et al., 1984). La presencia de concentraciones bajas de oxígeno ha sido mencionada como el principal factor limitante de la distribución de moluscos bénticos en el Golfo de California (Guerrero-Pelcastre, 1986) y en la plataforma continental de Guerrero, México (Lesser-Hiriart, 1984) 
Renteria. We would like to thank Manuel Guzmán-Arroyo and his group at the Instituto de Limnologia (UG) who gave us support during the oceanographic expedition Atlas $V$ on board the R/V El Puma. David Barrera (Instituto de Geografía, UG) made the determinations of the types of substratum found in the area of study. This manuscript was improved by the comments of two anonymous reviewers.

\section{REFERENCES}

Abbott. R.T. (1974). American Seashells. Van Nostrand Reinhold. New York. 663 pp.

Ambrogi, R., Beduili, D. and Zurlini, G. (1990). Spatial and temporal patterns in the structure of macrobenthic assemblages. A three year study in the northern Adriatic Sea in front of the Po River delta Mar. Biol., 1 I(1): 24-41

Buchanan, J.B., Sheader, M. and Kingston, P.F. (1978). Sources of variability in the benthic macrofauna of the south Northumberland coast, 1971-1976. J. Mar. Biol. Assoc. UK. 58: 191-209

Cifuentes-Lemus, J.L. (1986). Los moluscos como alimento actual y futuro. Mem. Il Reunión de Malacología. Villahermosa, Tabasco, México, pp. 123-154.

Draper. B.C. (1975). Checklist of shells collected at Cholla Bay, Sonora, Mexico. The Festivus, 6(11) 67.

DuShane. H, and Poorman, R. (1967). A checklist of mollusks of Guaymas, Sonora, Mexico. The Veliger, 9(4): 414-441.

Fagle, R.A. (1975). Natural fluctuations in a softbottom benthic community. J. Mar. Biol. Assoc. UK. 55: 865-878.

Frigiolos, N.A. and Zenetos, A. (1988). Elefsis Bay anoxia: nutrient conditions and bentlic community structure. Mar. Ecol., 9(4): 273-290.

Galaviz-Solís, A. y Gutiérrez-Estrada, M. (1978). Caracteristicas costeras y litorales de Nayarit y norte de Jalisco, México. Mem. VI Congreso Nacional de Oceanografia. Ensenada, Baja California. México, pp. 46-54

Garcia-Cubas. A.. Castillo-Rodriguez. Z.G.. AlvarezHerrera. A. y Muñoz-Chaguin, R. (1986). Moluscos comestibles de las costas de México. Mem. III Reunión Nacional de Malacología y Conquiliología. Monterrey. Nuevo León. México. pp. 43-56
Siete de las especies más abundantes en las muestras son carnivoros epifaunales, excepto $C$. perforans (Lindner, 1975). Las especies epifaunales tienen una mayor variedad de tallas y hábitos alimenticios que las especies infaunales, indicando una mayor radiación adaptativa. $\mathbf{L a}$ mayoría de los individuos de estas especies fueron capturados vivos en los tres tipos de substrato encontrados en el área de estudio. Estas especies constituyen un componente importante de la fauna de acompañamiento de las pesquerías comerciales de camarón en el Pacifico mexicano (García-Cubas et al., 1986). Estas especies son de tamaño considerable y son usadas como alimento, carnada y en artesanías locales (Cifuentes-Lemus, 1986).

\section{AGRADECIMIENTOS}

Esta investigación fue realizada con el apoyo del equipo de campo y laboratorio de la Universidad de Guadalajara (UG) y la Universidad Nacional Autónoma de México. Todas las personas del Laboratorio de Ecología Marina en la anterior Facultad de Ciencias (UG) ofrecieron gran ayuda durante el trabajo de campo y laboratorio, especialmente Ernesto López-Uriarte, Lucía Lizárraga y Samuel Rentería. Agradecemos a Manuel Guzmán-Arroyo y su grupo de trabajo del Instituto de Limnología (UG), quienes nos apoyaron durante la expedición oceanográfica Atlas $V$ a bordo del $\mathrm{B} / \mathrm{O}$ El Puma. David Barrera (Instituto de Geografía. UG) realizó las determinaciones de los tipos de substrato encontrados en el área de estudio. Este manuscrito fue mejorado por los comentarios de dos revisores anónimos.

Traducido al español por los autores.

Guerrero-Pelcastre, V.M. (1986). Sistemática y ecología de los moluscos bentónicos del Golfo de California. Tesis de licenciatura. Escuela Nacional de Estudios Profesionales. Iniversidad Nacional Autónoma de México. México. DF.

Guzmán-Arroyo. M. y Flores-Rosas. F. (1988). Campaña Oceanogrática Allas I Jalisco-Colima: 
Informe de actividades del Instituto de Limnología, Universidad de Guadalajara, Guadalajara, México, $9 \mathrm{pp}$.

Hendrickx, M.E., van der Heiden, M.N. y ToledanoGranados, ^. (1981). Resultados de las campañas SIPCO (sur de Sinaloa, México) a bordo del $\mathrm{B} / \mathrm{O}$ El Puma: Hidrología y composición de las capturas efectuadas en los arrastres. An. Inst. Cienc. Mar y Limnol., UNAM, 2(2): 107-122.

Herrera-Peña, J. (1981). Moluscos de la región del Golfo de California. Dir. Gral. Ocean. y Señal. Mar., Secretaria de Marina, México, DF, 35 pp.

Hertlein, L. and Allison, E. (1960). Gastropods from Clipperton Islands. The Veliger, 3(1): 13-15.

Holguín-Quiñones, O.E., Doval, L. y Flores, C. (1987). Algunas relaciones interespecíficas entre algas $y$ moluscos en facies rocosas de la franja de mareas del estado de Oaxaca, México. Mem. Soc. Mex. Malacol., 3: 91-105

Keen, M.^. (1971). Sea Shells of Tropical Western America. 2nd ed. Stanford Univ. Press, Stanford, California, $1064 \mathrm{pp}$.

Keen, M.A. and Coan, E. (1974). Marine Molluscan Genera of Western North America. 2nd ed. Stanford Univ. Press, Stanford, California, $208 \mathrm{pp}$.

Kiorbe, T. (1979). The distribution of benthic invertebrates in Holbaek Fjord (Denmark) in relation to environmental factors. Ophelia, 18(1): 6181 .

Lankford, R.R. (1977). Coastal lagoons of Mexico: their origin and classification. Mar. Geologist, $182-215$

Lesser-Hiriart, H. (1984). Prospección sistemática y ecológica de los moluscos bentónicos de la plataforma continental del estado de Guerrero, México. Tesis de licenciatura, Facultad de Ciencias, Universidad Nacional Autónoma de México, México, DF.

Lindner, G. (1975). Field Guide to Seashells of the World. Van Nostrand Reinhold, New York, $271 \mathrm{pp}$.

Morris, P.A. (1966). A Field Guide to Shells of the Pacific Coast and Hawaii. Houghton Mifflin, Boston, 297 pp.
Parker, R.H. (1964). Zoogeography and ecology of some macroinvertebrates, particularly mollusks, in the Gulf of California and the continental slope off Mexico. Videnskabelige Meddelelser fra Dansk Naturhistorisk Forening, 126: 1-178.

Parsons, T.R., Takahashi, M. and Hargrave, B. (1984). Biological Oceanographic Processes. 3rd ed. Pergamon Press, Elmsford, New York, 330 pp.

Pérez-Peña, M. (1994). El sistema bentónico sublitoral en la costa norte del Pacífico México-EUA: Campaña ECOBAC III-0690 (31 $1^{\circ} 30^{\prime}-32^{\circ} 45^{\prime}$ LN). Tesis de maestría, Centro de Investigación Científica y de Educación Superior de Ensenada, Baja California, México.

Reguero-Reza, M. (1985). Moluscos de la plataforma continental de Nayarit: Sistemática y ecología. Tesis de licenciatura, Facultad de Ciencias, Universidad Nacional Autónoma de México. México, DF.

Ríos-Jara, E., Pérez-Peña, M., Lizárraga-Chávez, L. and Michel-Morfïn, J.E. (1996). Additional gastropod records from the continental shelf off Jalisco and Colima, Mexico. Ciencias Marinas, 22(3): 347-359.

Ruíz-Durán, M.F. (1985). Recursos Pesqueros de las Costas de México. 2da ed. Limusa, México, DF, $135 \mathrm{pp}$

Sanders, H.L. (1968). Marine benthic diversity: a comparative study. Am. Nat., 102: 243-282.

Sanders, H.L. and Hessler, R.R. (1969). Ecology of the deep sea benthos Science, 163: 1419-1424.

Secretaria de Marina (1980). Estudio Oceanográfico del Golfo de Tehuantepec. Dir. Gral. Ocean. Señal. Mar., Secretaría de Marina, México, DF, 38 pp.

Skoglund, C. (1992). Additions to the Panamic Province gastropod (Mollusca) literature 1971-1992. The Festivus, XXIV (Suppl.), 169 pp.

Warwick, R.M. and Uncles, R.J. (1980). Distribution of benthic macrofauna associations in the Bristol Channel in relation to tidal stress. Mar. Ecol. Prog. Ser., 3: 97-103.

Wildish, D.J. (1977). Factors controlling marine and estuarine sublittoral macrofanna. Helgo. Wiss. Meers., 30: 445-454 